1 Hall J. Schizophrenia - an anxiety disorder? Br J Psychiatry 2017; 211: 262-3.

2 Baeza-Velasco C, Pailhez G, Bulbena A, Baghdadli A. Joint hypermobility and the heritable disorders of connective tissue: clinical and empirical evidence of links with psychiatry. Gen Hosp Psychiatry 2015; 37: 24-30.

3 Bowen JM, Sobey GJ, Burrows NP, Colombi M, Lavallee ME, Malfait F, et al. Ehlers-Danlos syndrome, classical type. Am J Med Genet C Semin Med Genet 2017; 175: 27-39.

4 Heikkinen A, Pihlajaniemi T, Faissner A, Yuzaki M. Neural ECM and synaptogenesis. Prog Brain Res 2014; 214: 29-51.

Jeremy Hall, Neuroscience and Mental Health Research Institute, Cardiff University, UK. Email: hallj10@cardiff.ac.uk

doi:10.1192/bjp.2018.127

\section{Personality disorder and suicide}

The discussion about the association between personality disorder and suicide is important. ${ }^{1}$ First, I want to present some information on the risk of suicide in patients with personality disorder with special reference to the subtype of personality disorder.

Coleman et al. conducted a cross-sectional study to understand the relationship between narcissistic personality disorder (NPD) and suicidal behaviour in 657 patients with mood disorders. ${ }^{2}$ The adjusted odds ratio for suicide attempt in the participants with NPD was 0.41 (95\% CI 0.19-0.88). In addition, being male, substance use disorder, aggression and hostility also presented a significant increase in odds ratio for suicide attempt. In contrast, the adjusted odds ratio for suicide attempt in those participants with borderline personality disorder (BPD) was 4.96 (95\% CI 3.25-7.58). NPD in patients with mood disorders showed a protective effect for suicidal behaviour, which was different from that in participants with BPD. Sher reports that patients with NPD have a risk of suicide behaviour, ${ }^{3}$ and I recommend stratified analysis by subtype of personality disorder for risk assessment of suicide.

Second, there is a difference between suicidal ideation and attempt. ${ }^{4}$ In addition, mood disorder is a risk factor for suicidal behaviour. ${ }^{5}$ Wang et al. conducted a prospective study to investigate the effect of stressful life events on subsequent suicidal behaviour in patients with major depressive disorder. ${ }^{6}$ They clarified that financial stress was a strong predictor for suicide attempts after adjusting for sociodemographic variables, anxiety, substance use and personality disorder. As Liu also pointed out, comprehensive analysis is recommended for risk assessment of suicide.

1 Liu KY. Personality disorders and suicide in China. Br J Psychiatry 2017; 210: 167

2 Coleman D, Lawrence R, Parekh A, Galfalvy H, Blasco-Fontecilla H, Brent DA, et al. narcissistic personality disorder and suicidal behavior in mood disorders. J Psychiatr Res 2017; 85: 24-8.

3 Sher L. Narcissistic personality disorder and suicide. Psychiatr Danub 2016; 28 : 307.

4 Lewitzka U, Spirling S, Ritter D, Smolka M, Goodday S, Bauer M, et al. Suicidal ideation vs. suicide attempts: clinical and psychosocial profile differences among depressed patients: a study on personality traits, psychopathological variables, and sociodemographic factors in 228 patients. J Nerv Ment Dis 2017; 205: 361-71.

5 Vilaplana M, Richard-Devantoy S, Turecki G, Jaafari N, Jollant F. Insight into mental disorders and suicidal behavior: a qualitative and quantitative multimodal investigation. J Clin Psychiatry 2015; 76: 303-18.

6 Wang Y, Sareen J, Afifi TO, Bolton SL, Johnson EA, Bolton JM. A populationbased longitudinal study of recent stressful life events as risk factors for suicidal behavior in major depressive disorder. Arch Suicide Res 2015; 19: 202-17.

Tomoyuki Kawada, Professor, Department of Hygiene and Public Health, Nippon Medical School, Japan. Email: kawada@nms.ac.jp

doi:10.1192/bjp.2018.133

\section{A letter to Jim Crabb et al}

Congratulations on your article, ${ }^{1}$ which I have been thinking about since reading it some weeks ago. I fully agree with your aims (attracting doctors into the specialty) and many of your arguments, but also have some reservations, which I would like to share. I am also wondering what kind of reactions you have had from others, and whether you have heard anything similar to the following observations.

In this age of internet 'click-bait', it seems, when advertisements are designed 'to create an anxiety relieved by a purchase', people are often naturally suspicious of advertising, branding and marketing. It is seldom fully truthful, misleading by presenting opinion as facts, by being selective of data, and by concealing flaws and inadequacies. This is the hype, spin or propaganda designed to sell products and maximise financial profit, and arguably therefore unsuitable for persuading medical students and young doctors to think about psychiatry as a long-term career path.

In the cold light of day, for example, particularly in today's evidence-based, politically governed, underfunded and overstretched National Health Service, people might wonder how truthful are the statements comprising the mantra you propose for 'brand psychiatry'. The experience of a patient, or family members, might not be exactly as you describe. The rational, materialist, left-brain dominated 'scientific' approach - which tends to search out symptoms and diagnoses, and then to provide physical treatments (medication) and brief impersonal psychotherapies (such as cognitive-behavioural therapy), rather than seeking healing for the whole person, body, mind and soul - still prevails over a more holistic, intuitive, poetic, right-brain dependent, person-oriented approach, do you not think?

I am sure, however, that you are on the right track. It is a genuine ideal to be pursued, 'To understand the connection between the mind, the body and the soul', and 'To have the rare ability to treat the person, not the problem'. But might not other doctors, particularly general practitioners, want to make similar claims?

An approach that might work well could be to stress the equal values of biological, psychological, social and spiritual aspects of mental healthcare (see for example, regarding the latter Culliford ${ }^{2,3}$ and Cook et $a l^{4}$ ). In other words, giving the message that there is a welcome in the specialty for people with a wide range of knowledge, skills and experience, enabling each to grow - through training and practice - in those areas and attributes perhaps previously less well developed.

There is an important place for those whose abilities and preferences lie within the biological domain, still the 'comfort-zone' for many psychiatrists; but the aim, I suggest, is both to encourage such folk to broaden their horizons out of their familiar orbit, and to encourage new people to enter the specialty whose inclinations are more towards (to paraphrase the mantra) 'feeling with one's mind' and 'thinking with one's soul'. Arguably, this means fostering awareness and familiarity with the spiritual dimension of mental healthcare. Some may be surprised to know that this valuable and hitherto neglected - aspect of our discipline can be taught. ${ }^{5}$

An axiom for this new psychiatry would be that everyone is on some kind of self-improvement pathway towards a maturity that involves personal integration, with continuing growth in terms of wisdom, compassion and love, derived from a sense of belonging not to any faction but to the entirety of humanity, similarly connected seamlessly to nature, to the dynamic structure and energy of the cosmos that underpin all the natural laws known to science. To become a psychiatrist would thus offer an unparalleled opportunity for making progress along this (I would call it 'spiritual') path. 
As you have hinted, personal sacrifices are necessary. It is a tough, and occasionally dangerous profession. It is not too hard academically, but it is challenging at a deeply personal level. Compassion - 'suffering with' one's patients and their carers, also one's colleagues - means feeling and sharing the emotional pain and distress of others. As I have written about extensively elsewhere, ${ }^{6}$ it is this very suffering, acting as a kind of medicine, which affords the best opportunity to initiate healing from life's inevitable psychological traumas, threats and losses, resulting in the deepest satisfaction that human experience can offer, inherent in personal growth. To become wiser, kinder, humbler, more truthful and tolerant, enjoying lower levels of anxiety, anger, sorrow, doubt, confusion, and greater levels of equanimity and selfesteem, accompanied most often by the heartfelt esteem of others, are among the inestimable rewards to be garnered. This is undoubtedly what I have gained from becoming and working as a psychiatrist.

1 Crabb J, Barber L, Masson N. Shrink rethink: rebranding psychiatry. Br J Psychiatry 2017; 211: 259-61.

2 Culliford L. Spiritual care and psychiatric treatment: an introduction Adv Psychiatr Treat 2002; 8: 249-61.

3 Culliford L. How to take a spiritual history. Adv Psychiatr Treat 2007; 13: 212-9.

4 Cook CCH, Powell A, Sims A. Spirituality and Psychiatry. RCPsych Publications, 2009.

5 Culliford L. Teaching spirituality and health care to 3 rd year medical students. Clin Teacher 2009; 6: 22-7.

6 Culliford L. Seeking Wisdom - A Spiritual Manifesto. Buckingham University Press, 2018.

Larry Culliford, Independent Author - Retired Consultant Psychiatrist, 18, Penlands Rise Steyning BN44 3PJ, West Sussex. Email: larry@ldc52.co.uk

doi:10.1192/bjp.2018.134

\section{Rethinking rebranding}

Recruiting sufficient psychiatrists in the UK apparently resists straightforward remedy. Crabb et al's recent editorial Shrink rethink: rebranding psychiatry is a welcome contribution on this subject. ${ }^{1}$ Innovative and provocative in turn, it urges that the psychiatric profession draw on expertise from the fields of advertising and public relations. We should engage with potential recruits by thinking of psychiatry as a 'brand'.

But brands are ethereal things. Their existence is championed by some, ${ }^{2}$ whereas others have written about the negative impact of brand-oriented corporate activity. ${ }^{3}$ Marketing psychiatry as a brand certainly has an attractive simplicity. Yet doing so situates the practice of psychiatry in the realm of things that are bought and sold, where it sits only uncomfortably.

The ubiquity of some brands is a marketing triumph, but emulating their tactics is not necessarily desirable. The advertising of brands seeks to sow discontent; to demonstrate to customers a gap in their life experience that a product can fill. Attempts to promote brands and products by association with certain desirable lifestyles may be effective, but also disingenuous. This approach may be acceptable for a soft drink but should be approached with caution by the medical profession.

In addition, the management priorities of the corporations that own many brands only faintly resemble psychiatry's governance structures. Psychiatry's relationship with its 'competitors' is more complex. If one company enters administration as a result of the crushing success of a rival, then that is capitalism 'working'. But if by increasing psychiatry's share of trainee recruitment we substantially weaken a fellow specialty, this success is equivocal.
Arguably, acknowledged or not, psychiatry is a brand of sorts. Doctors making career decisions may be accustomed to thinking of themselves as consumers and consider their options in a transactional way. In this case the explicit branding of psychiatry makes some sense, and in recognising this possibility Crabb et al provide a valuable insight. But promoting psychiatry as a brand may mean that other ways of understanding how our specialty might appeal are overlooked. What I hope is not lost is the notion of the new recruits to psychiatry's ranks as engaged citizens, drawn to this specialty as an expression of deeply held values and as a demonstration of commitment to their community and to wider society.

1 Crabb J, Barber L, Masson N. Shrink rethink: rebranding psychiatry. Br J Psychiatry 2017; 211: 259-61.

2 The Economist. The case for brands. The Economist 2001; 8 September

3 Klein N. No Logo (10th anniversary edn). Fourth Estate, 2010.

Stephen Ginn, Consultant Psychiatrist, Camden and Islington Foundation NHS Trust, UK. Email: stephen.ginn@candi.nhs.uk

doi:10.1192/bjp.2018.135

\section{Cognitive-behavioural therapy for chronic fatigue syndrome: neither efficacious nor safe}

Janse et al investigated the effect of two variants of internet-based cognitive-behavioural therapy (iCBT) for chronic fatigue syndrome (CFS): iCBT with protocol-driven feedback and iCBT with feedback on demand. ${ }^{1}$

First, it should be acknowledged that CBT trials for participants with CFS have a high preselection bias, i.e. self-selection, since, according to another study by two of the authors of Janse et al, patients seem to be sceptical about psychological interventions. ${ }^{2}$ Janse et al's study reported 'clinically relevant depressive symptoms' in both iCBT groups (protocol-driven feedback iCBT group 31\%, feedback-on-demand iCBT group 29\%), while depression and other psychological conditions that could explain 'chronic fatigue' exclude the diagnosis CFS. ${ }^{2}$ It is feasible that many patients who improved had depression, not CFS.

Comparing the number of patients working full-time in this study ${ }^{1}$ with other studies, for example Sunnquist et $a l^{3}$ the CFS (?) patients can be classified as 'mild cases'. ${ }^{2}$ Since CFS is a heterogeneous condition, the results of this study ${ }^{1}$ cannot be generalised to CFS.

Drop-out rates are not reported but the authors assumed a drop-out rate of $15 \%$ when deciding on sample size ${ }^{1}$ and other studies by the same group have reported even higher drop-out rates. ${ }^{2}$ They state 'a substantial number of patients did not fully adhere to the interventions.' ${ }^{1}$ One could also question whether accessing treatment modules and email contact are 'strict criteria' to guarantee adherence to the graded activity protocol. Although the authors state that 'The treatment is tailored to a patient's current activity pattern as assessed with actigraphy', (increased) activity levels was not included in the adherence criteria.

According to the authors, both iCBT conditions are efficacious, since $29 / 80(36 \%)$ in the protocol-driven feedback iCBT group and $34 / 80$ (43\%) in the feedback-on-demand iCBT achieved the 'normal range' for Checklist Individual Strength fatigue severity, compared with $12 / 80$ (15\%) in the waiting list group. ${ }^{1}$ However, the treatment effects of the protocol-driven feedback iCBT and feedback-on-demand iCBT in the study are by far insufficient to achieve 'normal levels of fatigue' (Checklist Individual Strength fatigue severity $\leq 27$ ) as defined in another study by two of the 